\title{
The effects of moderate energy restriction on apnoea severity and CVD risk factors in obese patients with obstructive sleep apnoea
}

\author{
Julia Freitas Rodrigues Fernandes ${ }^{1}$, Luciene da Silva Araújo ${ }^{1}$, Sergio Emanuel Kaiser ${ }^{1}$, \\ Antonio Felipe Sanjuliani ${ }^{1}$ and Márcia Regina Simas Torres Klein ${ }^{2 *}$ \\ ${ }^{1}$ Discipline of Clinical and Experimental Pathophysiology, Rio de Janeiro State University, CEP 20551-030, Rio de Janeiro, Brazil \\ ${ }^{2}$ Department of Applied Nutrition, Nutrition Institute, Rio de Janeiro State University, CEP 20559-900, Rio de Janeiro, Brazil
}

(Submitted 14 March 2015 - Final revision received 5 September 2015 - Accepted 10 September 2015-First published online 16 October 2015)

\section{Abstract}

Nutritional intervention for weight loss is one of the treatment options for obstructive sleep apnoea (OSA) in patients with overweight or obesity. However, the effects of moderate energy restriction on OSA severity are not yet known. The present study aimed to evaluate the effects of moderate energy restriction on OSA severity and CVD risk factors in obese patients with OSA. In this 16-week randomised clinical trial, twentyone obese subjects aged 20-55 years and presenting an apnoea/hypopnoea index (AHI) $\geq 5$ events/h were randomised into two groups: the energy restriction group (ERG) and the control group (CG). The ERG was instructed to follow an energy-restricted diet $-3347 \cdot 2 \mathrm{~kJ} / \mathrm{d}(-800 \mathrm{kcal} / \mathrm{d}$ ) and the CG was advised not to change their food intake. At the beginning and at the end of the study, participants underwent evaluation of the following: OSA (Watch-PAT200 ${ }^{\circledR}$ ), nutritional parameters, blood pressure, sympathetic activity, inflammatory biomarkers, metabolic profile and endothelial function. The ERG ( $n$ 11), compared with the CG ( $n$ 10), had a significantly greater reduction in body weight (Cohen's $d=-1 \cdot 19$; $P<0.001$ ), in AHI (Cohen's $d=-0.95 ; P=0.04$ ) and in plasma concentrations of adrenaline (Cohen's $d=-1 \cdot 02 ; P=0.04$ ) as well as a significantly greater increase in minimum $\mathrm{O}_{2}$ saturation (Cohen's $d=1.08 ; P=0.03$ ). Although energy restriction was not associated with significant improvements in CVD risk factors, medium-to-large effect sizes were observed, suggesting that the statistically non-significant difference between groups may be due to the small sample size. This study suggests that in obese patients with OSA, moderate energy restriction is able to reduce the parameters of OSA severity and sympathetic activity.

\section{Key words: Obstructive sleep apnoea: Weight loss: Obesity: Sympathetic activity: Metabolic profile: Endothelial function}

Obstructive sleep apnoea (OSA) is considered a public health problem due to its high prevalence in the general population ${ }^{(1)}$ and its association with both increased morbidity and mortality in the short term (traffic and workplace accidents) ${ }^{(2)}$ and in the long term $(\mathrm{CVD})^{(3-5)}$. The identification of the mechanisms responsible for the development of atherosclerosis triggered by OSA is of major importance, and despite significant efforts they remain incompletely understood $^{(6)}$. There is evidence that OSA, mainly driven by chronic intermittent hypoxia, can increase the risk for CVD through sustained sympathetic activation, oxidative stress, systemic inflammation, hypercoagulability, endothelial dysfunction and also by promoting metabolic abnormalities ${ }^{(6-8)}$.

Nutritional intervention for weight loss is one of the treatment options for OSA in patients with overweight or obesity, based on the knowledge that weight loss can improve the apnoea/ hypopnoea index (AHI) and oxygenation in such patients ${ }^{(9-12)}$. Several guidelines for the treatment of obesity recommend moderate energy restriction in order to achieve a modest weight loss of $5-10 \%$ in a 6 -month period ${ }^{(13-15)}$. However, the effects of this nutritional approach on the severity of OSA are not yet known ${ }^{(16)}$. To the best of our knowledge, all randomised clinical trials that have evaluated the impact of weight loss on OSA severity explored either severe energy restriction, the association of energy restriction with physical activity or bariatric surgery ${ }^{(9,17,18)}$. Moderate energy restriction was tested in a few studies, but they lacked a control group (CG) and participants were also instructed to increase their physical activity $^{(19,20)}$. Furthermore, moderate weight loss in patients with excess body weight is associated with significant improvement in different CVD risk factors in the general population, including reduction in blood pressure, improvement of insulin resistance and lipid profile ${ }^{(13,21)}$. However, to date, it is not yet known about the effects of weight loss upon these different CVD risk factors in obese individuals with OSA.

Therefore, the purpose of this study was to evaluate the effects of moderate energy restriction on OSA severity, sympathetic

Abbreviations: AHI, apnoea/hypopnoea index; CG, control group; ERG, energy restriction group; hs-CRP, high-sensitivity C-reactive protein; OSA, obstructive sleep apnoea; WC, waist circumference.

*Corresponding author: M. R. S. T. Klein, fax +55 212334 2063, email marciarsimas@gmail.com 
activity, blood pressure, inflammatory biomarkers, metabolic profile and endothelial function in obese patients with OSA.

\section{Methods}

The present study was conducted at the Laboratory of Clinical and Experimental Pathophysiology, CLINEX, located at Pedro Ernesto University Hospital, Rio de Janeiro State University. The present study was conducted in accordance with the Declaration of Helsinki, and all the procedures involving human subjects/patients were approved by the committee on ethics and research of the Pedro Ernesto University Hospital (3017/2011-CEP/HUPE-CAAE:0161.0.228.000-11). Written informed consent was obtained from all the patients. The present trial was registered at www.clinicaltrials.gov (ID no. NCT02206126).

The present study was a 16-week, parallel-group, open-label, randomised, clinical trial. The inclusion criteria were as follows: individuals with obesity (grade I or II), aged between 20 and 55 years and with $\mathrm{AHI} \geq 5$ events/h. Participants were enrolled between July 2011 and October 2013. Potential participants were recruited from the waiting rooms of the Departments of Orthopedics, Plastic Surgery and Gynecology at the Pedro Ernesto University Hospital.

Exclusion criteria were as follows: smoking; use of dietary supplements; use of medications that could interfere with body weight, metabolic profile and/or blood pressure; use of $\alpha$-adrenergic-blocking agents; use of a permanent pacemaker; recent changes in body weight defined as at least $>3 \mathrm{~kg}$ within the previous 6 months as well as recent modifications in dietary intake and/or intensity or frequency of physical exercise during the same period; diagnoses of diabetes mellitus, hypertension, dyslipidaemia (with drug treatment) and/or kidney disease; clinical history of thyroid dysfunction, angina pectoris, heart failure, myocardial infarction and/or stroke; presence of peripheral vascular disease, peripheral neuropathy, liver failure, chronic pulmonary disease and gastro-oesophageal reflux disease; finger deformity that prevents the proper use of the sensors necessary to evaluate OSA and endothelial function; and previous bilateral cervical-thoracic sympathectomy. Pregnant or lactating women were not allowed to participate in the study.

At the beginning of the study (week 0), potentially eligible subjects were scheduled to arrive at the Laboratory between 08.00 and 10.00 hours after a 12 -h fasting period. When fasting, they underwent evaluation of their nutritional status, blood pressure, sympathetic nervous system activity, inflammatory biomarkers, metabolism of glucose and lipids and endothelial function. Subsequently, the dietary intakes of the participants were evaluated and they received a portable device (WatchPAT200 ${ }^{\circledR}$; Itamar Medical) and all the necessary instructions to conduct the home sleep study.

The following day, after the sleep study, participants visited the laboratory and returned the Watch-PAT200 ${ }^{\circledR}$. During the same visit, participants who presented with $\mathrm{AHI} \geq 5$ events/h were randomised into two groups: the energy restriction group (ERG) and the CG. The ERG was instructed to follow an energy-restricted diet $-3347.2 \mathrm{~kJ} / \mathrm{d}(-800 \mathrm{kcal} / \mathrm{d})$ and the CG was advised not to change their food intake.

Subsequent visits were scheduled with 4-week intervals (weeks 4, 8, 12 and 16). All the participants underwent an evaluation of their dietary intake, anthropometric parameters and blood pressure at weeks 4, 8 and 12. At the end of the study (week 16), participants of both the groups underwent all the evaluations performed at week 0 , as previously described, and once again received the Watch-PAT200 ${ }^{\circledR}$ for another home sleep study.

Both the groups were instructed not to change their usual exercise routine during the study period. At weeks $0,4,8,12$ and 16, physical activity was evaluated using the Baecke questionnaire ${ }^{(22)}$. This questionnaire evaluates physical activity at three subscales: at work, sports during leisure time and other physical activities during leisure time ${ }^{(22,23)}$.

\section{Dietary intervention}

The energy intake of the participants allocated to the ERG was formulated to correspond to an $3347 \cdot 2 \mathrm{~kJ} / \mathrm{d}$ ( $800 \mathrm{kcal} / \mathrm{d}$ ) reduction in baseline total daily energy expenditure, calculated using the Dietary Reference Intake equations ${ }^{(24)}$. This energy-restricted diet was structured to provide levels of macronutrients as follows: $\pm 15-20 \%$ of energy from proteins, $\pm 25-30 \%$ of energy from fat and $\pm 50-60 \%$ of energy from carbohydrates. At weeks 4, 8 and 12, the total energy value of the diet was adjusted according to the participant's body weight at that time, as an attempt to continue weight loss.

The participants allocated to the CG were advised not to change their food intake. However, after the 16-week follow-up period, participants in the CG received nutritional counselling for energy restriction.

\section{Sleep study}

The diagnosis of OSA was made using the wrist-worn portable device Watch-PAT200 ${ }^{\circledR}$. This device, approved by the Food and Drug Administration, allows accurate and clinically effective home diagnosis of OSA, with a highly significant correlation between the AHI obtained from polysomnography ${ }^{(25,26)}$.

The Watch-PAT200 ${ }^{\circledR}$ is a six-channel non-invasive device. The channels of the device are as follows: (1) peripheral arterial tonometry (PAT), (2) pulse oximetry, (3) actigraphy, (4) heart rate, (5) body position and (6) snoring detection ${ }^{(26)}$. The PAT signal measures the finger arterial pulsatile volume changes that are regulated by $\alpha$-adrenergic innervation of the smooth muscles of the finger vasculature, and thus reflects sympathetic nervous system activity. This augmentation in sympathetic activity accompanies the increase in heart rate and $\mathrm{O}_{2}$ desaturation at the termination of respiratory events. Thus, the Watch-PAT200 ${ }^{\circledR}$ indirectly detects apnoea/hypopnoea events by identifying surges of sympathetic activation associated with the termination of such events ${ }^{(25)}$.

Following the sleep study, the recordings were automatically downloaded and analysed by an offline procedure using zzzPAT software. The zzzPAT algorithms use four Watch-PAT200 ${ }^{\circledR}$ channels for the detection of different parameters, including 
AHI, respiratory disturbance index (RDI), $\mathrm{O}_{2}$ desaturation index (ODI), and mean and minimum $\mathrm{O}_{2}$ saturation. AHI refers to the number of apnoeas and hypopnoeas per hour of sleep, and RDI includes both the $\mathrm{AHI}$ and the respiratory effort-related arousal (RERA) index together. RERA is an obstructive event that does not meet the criteria for apnoea or hypopnoea but causes arousal from sleep. ODI expresses the number of $\mathrm{O}_{2}$ desaturation events during an hour of sleep. A desaturation event is determined as a reduction of $4 \%$ or more of the oxygen saturation baseline level. In the present study, the diagnosis of OSA was considered positive when AHI was $\geq 5$.

\section{Nutrition assessment}

The usual dietary intake assessment was performed using a semi-quantitative FFQ at week 0 . This questionnaire could determine the usual daily intake of energy, proteins, carbohydrates, lipids, cholesterol and fibres over the previous 6 months. This questionnaire, containing eighty items and usual portions, was developed for the Brazilian population based on habitual consumption of food and was validated against more accurate methods ${ }^{(27)}$. Alcohol consumption was considered when the reported frequency was 1 or more times/week.

Dietary intake during the study (weeks 4, 8, 12 and 16) was assessed through a 3 -d food record, covering 2 weekdays and 1 weekend day. The food record was reviewed and clarified during an interview with a registered dietitian. In the ERG, the food record was used to assess dietary adherence. In the CG, the food record was used to assess whether the study participants maintained the baseline dietary pattern.

Height was measured using a stadiometer accurate to $\pm 0.5 \mathrm{~cm}$, and weight was measured using a digital scale accurate to $\pm 0 \cdot 1 \mathrm{~kg}$ (Filizola S.A.), with participants wearing light clothing and no shoes, and they also attempted to empty their bladder. BMI was calculated using the standard equation $\left(\mathrm{kg} / \mathrm{m}^{2}\right)$. Waist circumference (WC) was measured in the standing position midway between the lower margin of the last rib and the iliac crest at mid-exhalation. Waist:height ratio was obtained by dividing WC $(\mathrm{cm})$ by height $(\mathrm{cm})$. Neck circumference $(\mathrm{NC})$ was measured at the level of the cricothyroid membrane ${ }^{(28)}$. Anthropometric measurements were taken twice and mean values were used in all the analyses.

Percentage of body fat was estimated by electrical bioimpedance using a Biodynamics BIA-450 body fat analyser (Biodynamics Corp.).

\section{Laboratory parameters}

Blood samples were collected after a $30-$ min resting period in the supine position. Aliquots of plasma and serum were stored at -20 or $-70^{\circ} \mathrm{C}$ as appropriate for laboratory determinations. The blood sample assigned for the determination of plasma catecholamines was collected in a cold tube and plasma was separated in a refrigerated centrifuge. Laboratory parameters included fasting circulating levels of glucose, insulin, lipids, high-sensitivity C-reactive protein (hs-CRP), adiponectin, adrenaline, noradrenaline, dopamine, intercellular adhesion molecule 1 (ICAM-1) and P-selectin.
Fasting plasma glucose level was measured by an automated technique, glucose oxidase/peroxidase method, using a commercial kit (BioSystems). Fasting plasma insulin levels were determined by the ELISA method using the commercially available specific kit (EMD Millipore Corporation). Insulin resistance status was assessed using the homoeostasis model assessment of insulin resistance (HOMA-IR) index, which was calculated as fasting insulin $(\mu \mathrm{U} / \mathrm{ml}) \times$ fasting plasma glucose $(\mathrm{mmol} / \mathrm{l}) / 22 \cdot 5^{(29)}$.

Concentrations of total cholesterol, HDL-cholesterol and TAG were assessed by an automated technique. LDL-cholesterol was estimated using Friedewald's formula ${ }^{(30)}$.

To evaluate biomarkers of inflammatory state, circulating levels of hs-CRP and adiponectin were determined. The turbidimetry method was used to determine the serum concentration of hs-CRP (BioSystems). ELISA was used to assess plasma adiponectin concentrations.

The activity of the sympathetic nervous system was evaluated by determining plasma concentrations of adrenaline and noradrenaline measured by ELISA using a commercial kit (DIAsource ImmunoAssays S.A.). To evaluate endothelial function, circulating levels of ICAM-1 and P-selectin were determined by the luminex ${ }^{\mathrm{TM}}$ xMAP method using a commercial kit (EMD Millipore Corporation).

\section{Blood pressure}

Blood pressure was recorded using a calibrated and semi-automatic sphygmomanometer device, OMRON ${ }^{\circledR}$ Model HEM-742INT (Omron Healthcare), after a resting period of $10 \mathrm{~min}$. The first reading was discarded and the mean of three consecutive measurements, taken in the non-dominant arm with 3-min intervals between readings, was used for the study. An appropriate arm cuff was used, and patients were instructed to stay free from tight clothing, sit with their legs uncrossed, with their feet on the floor, back supported, arm supported at heart level, palm facing upwards and elbow slightly flexed ${ }^{(31)}$.

\section{Endothelial function}

Endothelial function was also evaluated by the PAT method, using Endo-PAT $2000^{\circledR}$, a finger plethysmographic device (Itamar Medical). This is a non-invasive method that offers the possibility of an easy and rapid assessment of vascular function in which data are analysed independently of the examiner ${ }^{(32)}$

The measurements were taken using fingertip probes placed on both index fingers. A 5-min measurement was taken at baseline. Sequentially, arterial flow was occluded using a cuff on the non-dominant arm, which was inflated to $60 \mathrm{mmHg}$ above systolic blood pressure, but never below $200 \mathrm{mmHg}$. The cuff was rapidly deflated after $5 \mathrm{~min}$ of occlusion to allow reactive hyperaemia, and the following $5 \mathrm{~min}$ were then recorded. The other arm served as a control and the difference between the two arms was used by the Endo-PAT $2000^{\circledR}$ software to automatically calculate the reactive hyperaemia index. 


\section{Statistical methods}

Means with their standard errors were used to summarise continuous variables. Normality was tested by using the Shapiro-Wilk's test. A Student's $t$ test or the Mann-Whitney test was used to assess the differences of variables between the two groups at week 0 and to evaluate the intragroup and intergroup differences for variables evaluated at week 0 and week 16 . Categorical variables were expressed as percentage and compared by the $\chi^{2}$ test. Repeated-measures ANOVA were used to evaluate intragroup and intergroup differences for anthropometric parameters and blood pressure levels (which were evaluated at weeks $0,4,8,12$ and 16). Cohen's $d$ effect size was calculated for the intergroup differences. As effect sizes are not dependent upon the sample size, they can help inform about the potentially meaningful effects of the intervention. Effect sizes of 0.20 are small, 0.50 are medium and 0.80 are large ${ }^{(33)}$.

Correlations between continuous variables were determined using the Pearson's or Spearman's correlation coefficient. Partial correlations adjusted for potential confounders (age, sex, values of the variables involved in the analysis at baseline, changes in body weight and changes in WC) were also used.

Statistical analyses were limited to those who completed the intervention ( $n$ 21). All the analyses were performed using
STATA (version 10.0; Stata Corp.) software. A $P$ value $<0.05$ was considered to be statistically significant. Randomisation was performed in blocks.

It was not possible to determine the desired sample size to achieve a significant decrease in AHI, due to the non-existence of a previous randomised clinical trial with a similar intervention.

\section{Results}

A total of 266 subjects were interviewed, of which thirty-three met the eligibility criteria and twenty-nine were randomised. The most common reasons for exclusion were smoking ( $n$ 30), hypertension ( $n$ 41), BMI $<30 \mathrm{~kg} / \mathrm{m}^{2}$ ( $n$ 40), BMI $\geq 40 \mathrm{~kg} / \mathrm{m}^{2}$ ( $n$ 13), age $>55$ years $(n 8)$ and diabetes $(n 5)$. Among randomised subjects ( $n$ 29), twenty-one completed the study, with eleven participants in the ERG and ten in the CG (Fig. 1). The non-completers left the study because of lack of interest, personal reasons and changes in work schedule.

Both the study groups were comparable in all parameters evaluated at baseline (week 0) (Table 1). Compliance to dietary prescription in the ERG and maintenance of the habitual dietary intake during the study period in the CG were satisfactory (Table 2). Participants maintained their physical activity during the study period (Table 2).

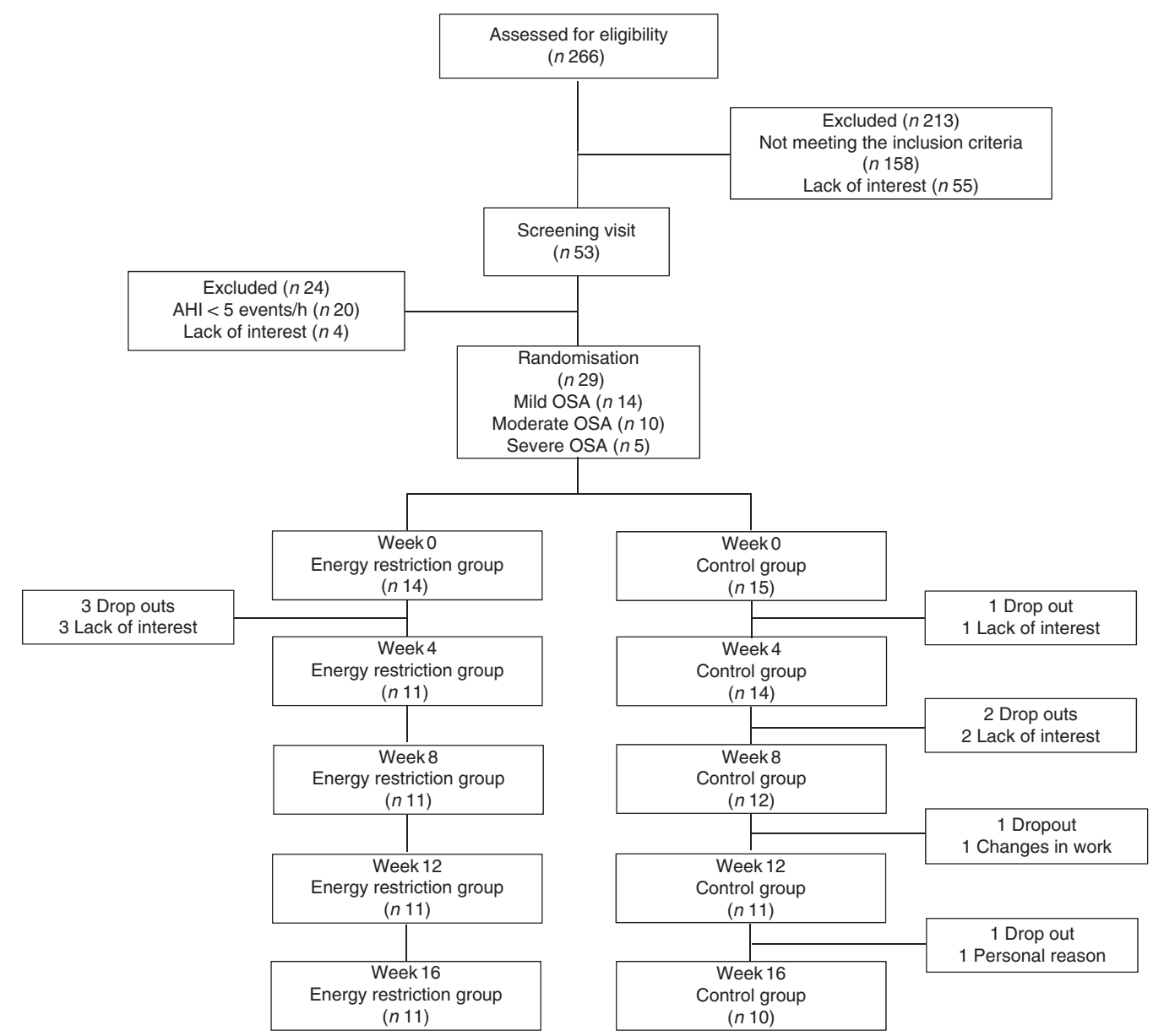

Fig. 1. Flow diagram of the study. AHI, apnoea/hypopnoea index; OSA, obstructive sleep apnoea. 
Table 1. Baseline characteristics of study subjects

(Mean values with their standard errors)

\begin{tabular}{|c|c|c|c|c|c|}
\hline \multirow[b]{2}{*}{ Characteristics } & \multicolumn{2}{|c|}{ Energy restriction group $(n 11)$} & \multicolumn{2}{|c|}{$\begin{array}{l}\text { Control group } \\
\quad(n-10)\end{array}$} & \multirow[b]{2}{*}{$P^{*}$} \\
\hline & Mean & SE & Mean & SE & \\
\hline Age (years) & 39.09 & $3 \cdot 26$ & $44 \cdot 10$ & 1.95 & 0.21 \\
\hline Sex (female/male) & $4(36 \%) / 7(64 \%)$ & & $6(60 \%) / 4(40 \%)$ & & 0.28 \\
\hline \multicolumn{6}{|l|}{ Sleep study } \\
\hline Apnoea/hypopnoea index (events/h) & $26 \cdot 67$ & 9.41 & $16 \cdot 88$ & 2.77 & 0.94 \\
\hline Minimum $\mathrm{O}_{2}$ saturation (\%) & $81 \cdot 10$ & $2 \cdot 51$ & 84.50 & 1.97 & 0.38 \\
\hline \multicolumn{6}{|l|}{ Nutrition assessment } \\
\hline Body weight (kg) & $100 \cdot 08$ & $4 \cdot 10$ & 99.73 & 4.49 & 0.95 \\
\hline $\mathrm{BMI}\left(\mathrm{kg} / \mathrm{m}^{2}\right)$ & 34.60 & 0.80 & 35.92 & 0.91 & 0.29 \\
\hline Body fat (kg) & $34 \cdot 64$ & 1.28 & $36 \cdot 29$ & 1.97 & 0.49 \\
\hline Body fat (\%) & 34.90 & 1.35 & 36.63 & 1.80 & 0.44 \\
\hline Waist circumference $(\mathrm{cm})$ & $105 \cdot 50$ & $2 \cdot 78$ & 109.83 & 2.96 & 0.30 \\
\hline Waist:height ratio & 0.62 & 0.01 & 0.66 & 0.02 & 0.08 \\
\hline Neck circumference $(\mathrm{cm})$ & $40 \cdot 32$ & 1.37 & $40 \cdot 74$ & 1.38 & 0.83 \\
\hline \multicolumn{6}{|l|}{ Usual dietary intake } \\
\hline Energy (kJ/d) & $9860 \cdot 80$ & $442 \cdot 96$ & 9323.50 & $927 \cdot 38$ & 0.59 \\
\hline Energy $(\mathrm{kcal} / \mathrm{d})$ & $2356 \cdot 79$ & $105 \cdot 87$ & 2228.37 & 221.65 & 0.59 \\
\hline Protein (g/d) & 77.98 & 3.88 & $70 \cdot 29$ & 5.84 & 0.28 \\
\hline Carbohydrates (g/d) & 332.05 & $17 \cdot 78$ & 323.52 & 41.00 & 0.84 \\
\hline Lipids (g/d) & 79.63 & 5.45 & $72 \cdot 57$ & $6 \cdot 82$ & 0.44 \\
\hline Cholesterol $(\mathrm{mg} / \mathrm{d})$ & $336 \cdot 50$ & $42 \cdot 81$ & 304.77 & 42.52 & 0.06 \\
\hline Fibre $(g / d)$ & 17.90 & 1.29 & $15 \cdot 26$ & $2 \cdot 27$ & 0.29 \\
\hline \multicolumn{6}{|l|}{ Usual physical activity } \\
\hline Work index & 3.59 & 0.60 & 2.93 & 0.41 & 0.38 \\
\hline Sport index & $2 \cdot 11$ & $0 \cdot 11$ & 2.55 & 0.24 & 0.10 \\
\hline Leisure-time index & 2.04 & 0.22 & $2 \cdot 25$ & 0.20 & 0.49 \\
\hline Total & 7.75 & 0.73 & 7.72 & 0.49 & 0.97 \\
\hline \multicolumn{6}{|l|}{ Laboratory parameters } \\
\hline Glucose $(\mathrm{mmol} / \mathrm{l})$ & 4.86 & 0.14 & $5 \cdot 27$ & 0.20 & 0.10 \\
\hline Urea $(\mathrm{mmol} / \mathrm{l})$ & $5 \cdot 19$ & 0.30 & 4.49 & 0.42 & 0.18 \\
\hline Creatinine (mg/dl) & 73.37 & 3.54 & 68.95 & 5.30 & 0.52 \\
\hline Total cholesterol $(\mathrm{mmol} / \mathrm{l})$ & 5.37 & 0.30 & 5.36 & 0.24 & 0.98 \\
\hline HDL-cholesterol $(\mathrm{mmol} / \mathrm{l})$ & 1.22 & $0 \cdot 13$ & $1 \cdot 28$ & 0.07 & 0.69 \\
\hline LDL-cholesterol (mmol/l) & 3.52 & 0.24 & 3.45 & 0.22 & 0.83 \\
\hline TAG $(\mathrm{mmol} / \mathrm{l})$ & 1.37 & 0.15 & 1.37 & 0.15 & 0.98 \\
\hline hs-CRP (mg/l) & $7 \cdot 1$ & $2 \cdot 1$ & $5 \cdot 3$ & $2 \cdot 1$ & 0.56 \\
\hline \multicolumn{6}{|l|}{ Blood pressure } \\
\hline Systolic blood pressure (mmHg) & 126.45 & $2 \cdot 88$ & 121.37 & 2.93 & 0.23 \\
\hline Diastolic blood pressure $(\mathrm{mmHg})$ & 84.24 & $2 \cdot 37$ & $81 \cdot 24$ & $2 \cdot 21$ & 0.47 \\
\hline \multicolumn{6}{|l|}{ Endothelial function } \\
\hline Reactive hyperaemia index & 1.82 & 0.09 & 1.96 & 0.12 & 0.44 \\
\hline
\end{tabular}

hs-CRP, high-sensitivity $C$ reactive protein.

* $P$ values refer to differences between the energy restriction group and the control group.

Participants allocated to the ERG, compared with those in the CG, presented a significant reduction in body fat and in all anthropometric parameters, as expected, due to the energy deficit in this group (Table 3). AHI decreased significantly in the ERG compared with the CG, whereas minimum $\mathrm{O}_{2}$ saturation increased significantly in the ERG compared with the CG (Table 4).

Subjects in the ERG compared with those in the CG showed significantly greater reduction in plasma levels of adrenaline. There was no significant difference between the groups in relation to the modifications in plasma insulin, HOMA-IR, hs-CRP, lipid profile, endothelial function and blood pressure (Table 5). However, medium-to-large effect sizes were observed for insulin, HOMA-IR, hs-CRP, adiponectin, ICAM-1 and blood pressure, suggesting that the statistically non-significant difference between the groups might be due to the small sample size.
During the study period, the modifications in body weight, body fat, WC and NC exhibited a significant correlation with changes in parameters of OSA severity, blood pressure, insulin levels and HOMA-IR, even after adjustments for confounding factors. The changes in WC were significantly associated with modifications in the levels of noradrenaline and adiponectin (Table 6).

Correlations between changes in variables obtained during the sleep study and the modifications in the biochemical biomarkers, blood pressure and endothelial function are presented in Table 7. The changes in parameters of OSA severity presented a significant association with the modifications in serum levels of hs-CRP. This association was independent of confounding factors (age, sex, changes in body weight and changes in WC). The changes in minimum $\mathrm{O}_{2}$ saturation were associated positively and significantly with changes in serum 
Table 2. Dietary intake and physical activity of the participants in the different groups of the study (Mean values with their standard errors)

\begin{tabular}{|c|c|c|c|c|c|c|c|c|c|c|}
\hline & \multirow[b]{2}{*}{ Mean } & \multirow[b]{2}{*}{ SE } & \multicolumn{2}{|c|}{ Week 4} & \multicolumn{2}{|c|}{ Week 8} & \multicolumn{2}{|c|}{ Week 12} & \multicolumn{2}{|c|}{ Week 16} \\
\hline & & & Mean & SE & Mean & SE & Mean & SE & Mean & SE \\
\hline \multicolumn{11}{|c|}{ Energy restriction group ( $n$ 11) } \\
\hline Dietary intake & \multicolumn{2}{|c|}{ Prescribed diet } & & & & & & & & \\
\hline Energy (kJ/d) & $7770 \cdot 48$ & 519.90 & $7827 \cdot 84$ & 482.91 & $7706 \cdot 34$ & $496 \cdot 18$ & $7820 \cdot 50$ & $520 \cdot 74$ & $7790 \cdot 61$ & $504 \cdot 21$ \\
\hline Energy (kcal/d) & $1857 \cdot 19$ & $124 \cdot 26$ & $1870 \cdot 90$ & $115 \cdot 42$ & $1841 \cdot 86$ & $118 \cdot 59$ & $1869 \cdot 13$ & 124.46 & $1862 \cdot 27$ & $120 \cdot 51$ \\
\hline Protein $(g / d)$ & $97 \cdot 30$ & $6 \cdot 40$ & $86 \cdot 28$ & 5.80 & 88.46 & 5.78 & $87 \cdot 85$ & $5 \cdot 34$ & $87 \cdot 68$ & $5 \cdot 13$ \\
\hline Carbohydrates (g/d) & $242 \cdot 19$ & $17 \cdot 54$ & $246 \cdot 76$ & $12 \cdot 79$ & $236 \cdot 15$ & $14 \cdot 78$ & 241.62 & $16 \cdot 86$ & $246 \cdot 15$ & $16 \cdot 34$ \\
\hline Lipids (g/d) & 55.47 & 3.29 & $59 \cdot 86$ & $17 \cdot 90$ & $60 \cdot 38$ & 4.62 & $61 \cdot 25$ & $4 \cdot 20$ & $58 \cdot 55$ & $4 \cdot 26$ \\
\hline Cholesterol (mg/d) & $186 \cdot 4$ & $15 \cdot 51$ & 194.68 & $17 \cdot 89$ & $207 \cdot 76$ & $12 \cdot 89$ & $212 \cdot 91$ & $15 \cdot 66$ & $196 \cdot 26$ & $13 \cdot 39$ \\
\hline Fibre $(g / d)$ & $24 \cdot 19$ & 1.46 & $35 \cdot 38$ & 1.77 & 32.03 & $2 \cdot 34$ & 31.61 & $2 \cdot 84$ & $36 \cdot 88$ & $4 \cdot 72$ \\
\hline Physical activity & \multicolumn{2}{|c|}{ Baseline } & & & & & & & & \\
\hline Work index & 3.59 & 0.60 & 3.62 & 0.59 & 3.54 & 0.56 & 3.63 & 0.58 & 3.58 & 0.60 \\
\hline Sport index & $2 \cdot 11$ & 0.11 & $2 \cdot 10$ & 0.09 & $2 \cdot 13$ & 0.12 & $2 \cdot 08$ & 0.07 & $2 \cdot 13$ & 0.10 \\
\hline Leisure-time index & 2.04 & 0.22 & 2.03 & 0.20 & $2 \cdot 06$ & 0.21 & 2.05 & 0.22 & 2.03 & 0.21 \\
\hline Total & $7 \cdot 75$ & 0.73 & $7 \cdot 75$ & 0.72 & $7 \cdot 73$ & 0.71 & $7 \cdot 76$ & 0.74 & $7 \cdot 74$ & 0.72 \\
\hline \multicolumn{11}{|l|}{ Control group (n 10) } \\
\hline Dietary intake & \multicolumn{2}{|c|}{ Prescribed diet } & & & & & & & & \\
\hline Energy (kJ/d) & \multicolumn{2}{|c|}{-} & 9080.70 & $1061 \cdot 35$ & $9422 \cdot 03$ & 1134.57 & $9697 \cdot 21$ & 442.95 & $9433 \cdot 20$ & 921.99 \\
\hline Energy (kcal/d) & \multicolumn{2}{|c|}{-} & $2170 \cdot 34$ & 253.67 & 2251.92 & $271 \cdot 17$ & 2317.69 & $105 \cdot 87$ & 2254.59 & $220 \cdot 36$ \\
\hline Protein (g/d) & \multicolumn{2}{|c|}{-} & $65 \cdot 36$ & 7.54 & $74 \cdot 34$ & $9 \cdot 37$ & 72.65 & 3.88 & 73.54 & $5 \cdot 77$ \\
\hline Carbohydrates (g/d) & \multicolumn{2}{|c|}{-} & $317 \cdot 52$ & $37 \cdot 12$ & $336 \cdot 04$ & 38.41 & $347 \cdot 00$ & $17 \cdot 78$ & $333 \cdot 62$ & 25.03 \\
\hline Lipids (g/d) & \multicolumn{2}{|c|}{-} & 70.98 & 4.99 & $67 \cdot 824$ & $11 \cdot 1$ & 71.01 & 5.45 & $69 \cdot 55$ & $9 \cdot 45$ \\
\hline Cholesterol (mg/d) & \multicolumn{2}{|c|}{-} & 281.51 & 49.88 & $252 \cdot 82$ & $46 \cdot 52$ & $325 \cdot 27$ & $42 \cdot 81$ & $306 \cdot 37$ & 33.99 \\
\hline Fibre (g/d) & \multicolumn{2}{|c|}{-} & $10 \cdot 69$ & 3.33 & $18 \cdot 79$ & $2 \cdot 83$ & $12 \cdot 66$ & 1.29 & $17 \cdot 18$ & $4 \cdot 84$ \\
\hline Physical activity & \multicolumn{2}{|c|}{ Baseline } & & & & & & & & \\
\hline Work index & 2.93 & 0.41 & 2.95 & 0.40 & 2.92 & 0.42 & 2.90 & 0.41 & 2.96 & 0.43 \\
\hline Sport index & 2.55 & 0.24 & 2.54 & 0.22 & 2.53 & 0.25 & 2.58 & 0.20 & $2 \cdot 51$ & 0.22 \\
\hline Leisure-time index & $2 \cdot 25$ & 0.20 & $2 \cdot 27$ & 0.21 & $2 \cdot 28$ & 0.22 & $2 \cdot 26$ & 0.21 & $2 \cdot 24$ & 0.22 \\
\hline Total & $7 \cdot 72$ & 0.49 & $7 \cdot 72$ & 0.49 & $7 \cdot 72$ & 0.49 & $7 \cdot 72$ & 0.49 & $7 \cdot 72$ & 0.49 \\
\hline
\end{tabular}

Table 3. Nutritional parameters at baseline (week 0) and at the end of the study (week 16) in the different groups of the study (Mean values with their standard errors)

\begin{tabular}{|c|c|c|c|c|c|c|c|c|c|c|c|c|c|c|}
\hline & \multicolumn{6}{|c|}{ Energy restriction group ( $n 11)$} & \multicolumn{6}{|c|}{ Control group ( $n$ 10) } & \multirow[b]{3}{*}{$P+$} & \multirow[b]{3}{*}{$d \neq$} \\
\hline & \multicolumn{2}{|c|}{ Week 0} & \multicolumn{2}{|c|}{ Week 16} & \multicolumn{2}{|l|}{$\Delta$} & \multicolumn{2}{|c|}{ Week 0} & \multicolumn{2}{|c|}{ Week 16} & \multicolumn{2}{|c|}{$\Delta$} & & \\
\hline & Mean & SE & Mean & SE & Mean & SE & Mean & SE & Mean & SE & Mean & SE & & \\
\hline Body weight (kg) & $100 \cdot 08$ & $4 \cdot 10$ & 94.51 & 3.60 & $-5 \cdot 57^{\star \star}$ & $1 \cdot 81$ & $99 \cdot 73$ & 4.49 & $100 \cdot 16$ & $5 \cdot 16$ & 0.43 & $1 \cdot 21$ & $<0.001$ & $-1 \cdot 191$ \\
\hline BMI $\left(\mathrm{kg} / \mathrm{m}^{2}\right)$ & 34.60 & 0.80 & 32.73 & 0.84 & $-1.87^{\star \star}$ & 0.59 & 35.92 & 0.91 & 36.03 & 1.09 & $0 \cdot 10$ & 0.45 & $<0.001$ & $-1 \cdot 151$ \\
\hline Body fat (kg) & 34.64 & $1 \cdot 28$ & 30.52 & $1 \cdot 39$ & $-4 \cdot 13^{\star}$ & 1.35 & $36 \cdot 29$ & 1.97 & $37 \cdot 33$ & 2.41 & 1.04 & 0.70 & 0.004 & -1.463 \\
\hline Body fat (\%) & 34.90 & 1.35 & 32.67 & 1.78 & $-2 \cdot 23^{*}$ & 0.82 & 36.63 & 1.80 & $37 \cdot 40$ & 1.86 & 0.77 & 0.36 & 0.004 & -1.438 \\
\hline Waist circumference (cm) & 105.5 & 2.78 & $100 \cdot 65$ & $3 \cdot 22$ & $-4 \cdot 85^{\star \star}$ & 1.57 & $109 \cdot 83$ & 2.96 & $111 \cdot 41$ & $3 \cdot 33$ & 1.58 & 0.96 & $<0.001$ & -1.588 \\
\hline WheiR & 0.62 & 0.01 & 0.59 & 0.02 & $-0.03^{\star *}$ & 0.01 & 0.66 & 0.02 & 0.67 & 0.02 & 0.01 & 0.01 & $<0.001$ & $-1 \cdot 234$ \\
\hline Neck circumference (cm) & $40 \cdot 32$ & $1 \cdot 37$ & $39 \cdot 45$ & 1.32 & $-0.86^{\star \star}$ & 0.29 & $40 \cdot 74$ & $1 \cdot 38$ & 40.68 & 1.35 & -0.06 & 0.22 & 0.008 & -0.953 \\
\hline
\end{tabular}

$\triangle$, Week 16-week 0; WheiR, waist:height ratio.

* $P$ value $<0.05$ for modification during the study in the energy restriction group.

** $P$ value $<0.001$ for modification during the study in the energy restriction group.

$\dagger P$ values refer to differences between the energy restriction group and the control group.

$\ddagger$ Cohen's $d$ effect size.

adiponectin levels. This association was attenuated and was no longer statistically significant $(P=0.06)$ after adjustment for the same confounding factors.

\section{Discussion}

The guidelines for the management of OSA recommend weight loss for all overweight patients ${ }^{(11,12)}$. However, evidence is still lacking to support the concept that modest weight loss, induced by moderate energy restriction, can represent a therapeutic strategy for OSA.

In the present study, modest weight loss, induced by moderate energy restriction, was able to significantly improve parameters of OSA severity (reduction in AHI and increase in minimum $\mathrm{O}_{2}$ saturation) ( $n$ 21). This finding is in agreement with other randomised ${ }^{(9,17,18)}$ and non-randomised ${ }^{(19,20,34-36)}$ trials in which weight loss, induced by different strategies, was associated with improvements in OSA severity. In contrast, 
Table 4. Obstructive sleep apnoea severity parameters at baseline (week 0 ) and at the end of the study (week 16) in the different groups of the study (Mean values with their standard errors)

\begin{tabular}{|c|c|c|c|c|c|c|c|c|c|c|c|c|c|c|}
\hline & \multicolumn{6}{|c|}{ Energy restriction group ( $n$ 11) } & \multicolumn{6}{|c|}{ Control group $(n 10)$} & \multirow[b]{3}{*}{$P+$} & \multirow[b]{3}{*}{$d \neq$} \\
\hline & \multicolumn{2}{|c|}{ Week 0} & \multicolumn{2}{|c|}{ Week 16} & \multicolumn{2}{|c|}{$\Delta$} & \multicolumn{2}{|c|}{ Week 0} & \multicolumn{2}{|c|}{ Week 16} & \multicolumn{2}{|c|}{$\Delta$} & & \\
\hline & Mean & SE & Mean & SE & Mean & SE & Mean & SE & Mean & SE & Mean & SE & & \\
\hline RDI (events/h) & 31.97 & 8.64 & $24 \cdot 76$ & 7.44 & $-7 \cdot 21^{*}$ & 3.07 & 21.42 & $2 \cdot 40$ & $21 \cdot 62$ & $2 \cdot 97$ & 0.20 & 1.63 & 0.11 & -0.918 \\
\hline $\mathrm{AHI}$ (events/h) & $26 \cdot 67$ & $19 \cdot 41$ & $19 \cdot 45$ & 8.03 & $-7 \cdot 22^{*}$ & $2 \cdot 79$ & $16 \cdot 88$ & $2 \cdot 72$ & $17 \cdot 01$ & 3.49 & 0.13 & 1.88 & 0.04 & -0.945 \\
\hline ODI (events/h) & $19 \cdot 36$ & 8.79 & 12.96 & 6.78 & $-6 \cdot 40^{*}$ & $2 \cdot 89$ & 9.09 & 1.69 & $9 \cdot 23$ & $2 \cdot 01$ & 0.14 & $1 \cdot 11$ & $0 \cdot 11$ & -0.905 \\
\hline Minimum $\mathrm{O}_{2}$ saturation (\%) & $81 \cdot 10$ & $2 \cdot 51$ & $85 \cdot 70$ & $1 \cdot 78$ & $4 \cdot 60^{*}$ & 1.55 & 84.5 & 1.97 & 83.9 & 2.59 & -0.60 & 1.42 & 0.03 & 1.078 \\
\hline Mean $\mathrm{O}_{2}$ saturation (\%) & $93 \cdot 10$ & 1.03 & $94 \cdot 10$ & $0 \cdot 71$ & 1.00 & 0.52 & $94 \cdot 70$ & 0.47 & $95 \cdot 20$ & 0.53 & 0.50 & 0.31 & 0.73 & 0.356 \\
\hline
\end{tabular}

$\triangle$, Week 16-week 0; RDI, respiratory disturbance index; $\mathrm{AHI}$, apnoea/hypopnoea index; $\mathrm{ODI}, \mathrm{O}_{2}$ desaturation index.

${ }^{\star} P$ value $<0.05$ for modification during the study (week $0 \mathrm{v}$. week 16 ) in each group.

$\dagger P$ values refer to differences between the energy restriction group and the control group.

$\ddagger$ Cohen's $d$ effect size.

Table 5. Biochemical variables, reactive hyperaemia index (RHI) and blood pressure (BP) at baseline (week 0 ) and at the end of the study (week 16$)$ in the different groups of the study

(Mean values with their standard errors)

\begin{tabular}{|c|c|c|c|c|c|c|c|c|c|c|c|c|c|c|}
\hline & \multicolumn{6}{|c|}{ Energy restriction group ( $n$ 11) } & \multicolumn{6}{|c|}{ Control group $(n 10)$} & \multirow[b]{3}{*}{$P \dagger$} & \multirow[b]{3}{*}{$d \neq$} \\
\hline & \multicolumn{2}{|c|}{ Week 0} & \multicolumn{2}{|c|}{ Week 16} & \multicolumn{2}{|l|}{$\Delta$} & \multicolumn{2}{|c|}{ Week 0} & \multicolumn{2}{|c|}{ Week 16} & \multicolumn{2}{|c|}{$\Delta$} & & \\
\hline & Mean & $\mathrm{SE}$ & Mean & SE & Mean & SE & Mean & SE & Mean & SE & Mean & SE & & \\
\hline \multicolumn{15}{|l|}{ Biochemical variables } \\
\hline Glucose $(\mathrm{mmol} / \mathrm{l})$ & 4.86 & 0.14 & 4.89 & 0.15 & 0.02 & $0 \cdot 16$ & $5 \cdot 27$ & 0.20 & 5.34 & 0.29 & 0.07 & 0.21 & 0.88 & -0.070 \\
\hline Insulin (mcU/ml) & $15 \cdot 30$ & 1.91 & $10 \cdot 20$ & 1.05 & $-5 \cdot 11^{\star}$ & 1.93 & 9.98 & 1.01 & 9.33 & 1.14 & -0.65 & 1.28 & 0.07 & -0.868 \\
\hline HOMA-IR & 3.39 & 0.51 & $2 \cdot 24$ & 0.26 & $-1 \cdot 15^{\star}$ & 0.49 & $2 \cdot 29$ & 0.20 & $2 \cdot 21$ & 0.29 & -0.08 & 0.33 & 0.09 & -0.783 \\
\hline Total cholesterol $(\mathrm{mmol} / \mathrm{l})$ & 5.37 & 0.30 & 5.03 & 0.22 & -0.34 & 0.18 & $5 \cdot 36$ & 0.24 & $5 \cdot 15$ & 0.25 & -0.21 & 0.13 & 0.56 & -0.263 \\
\hline HDL-cholesterol $(\mathrm{mmol} / \mathrm{l})$ & 1.22 & 0.13 & 1.15 & 0.10 & -0.07 & 0.06 & 1.28 & 0.07 & 1.22 & 0.08 & -0.06 & 0.02 & 0.48 & -0.040 \\
\hline LDL-cholesterol (mmol/l) & 3.52 & 0.24 & $3 \cdot 28$ & 0.17 & -0.24 & $0 \cdot 14$ & 3.45 & 0.22 & 3.32 & 0.22 & -0.13 & 0.11 & 0.71 & -0.248 \\
\hline TAG (mmol/l) & 1.37 & 0.15 & 1.31 & 0.12 & -0.06 & 0.16 & 1.37 & 0.15 & 1.34 & 0.16 & -0.02 & 0.09 & 0.83 & -0.096 \\
\hline \multicolumn{15}{|l|}{ Inflammatory biomarkers } \\
\hline hs-CRP (mg/dl) & 0.71 & 0.21 & 0.35 & 0.10 & $-0.36^{\star}$ & $0 \cdot 18$ & 0.53 & 0.21 & 0.44 & 0.26 & -0.09 & 0.08 & 0.30 & -0.589 \\
\hline Adiponectin $(\mu \mathrm{g} / \mathrm{ml})$ & 5.59 & 0.75 & 7.71 & 1.09 & $2 \cdot 12$ & 0.93 & $6 \cdot 67$ & 0.98 & 6.92 & 1.26 & 0.25 & 1.02 & 0.19 & 0.613 \\
\hline \multicolumn{15}{|l|}{ Plasma catecholamines } \\
\hline Adrenaline (pg/ml) & $18 \cdot 00$ & 3.60 & $5 \cdot 30$ & 2.00 & $-12 \cdot 70^{*}$ & 3.00 & 9.60 & 3.00 & $8 \cdot 30$ & 2.00 & -1.30 & 3.90 & 0.04 & -1.017 \\
\hline Noradrenaline $(\mathrm{pg} / \mathrm{ml})$ & 171.00 & $29 \cdot 00$ & 85.00 & $17 \cdot 00$ & $-86 \cdot 00^{*}$ & $28 \cdot 0$ & 189.00 & $50 \cdot 00$ & $164 \cdot 0$ & $40 \cdot 0$ & $-25 \cdot 0$ & $50 \cdot 0$ & 0.30 & -0.471 \\
\hline \multicolumn{15}{|l|}{ Endothelial function } \\
\hline $\mathrm{RHI}$ & 1.82 & 0.09 & 1.92 & 0.20 & $0 \cdot 10$ & 0.14 & 1.96 & 0.12 & 1.92 & 0.10 & -0.04 & 0.11 & 0.45 & 0.341 \\
\hline ICAM-1 $(n$ & $120 \cdot 63$ & $12 \cdot 08$ & $117 \cdot 31$ & $14 \cdot 11$ & -3.32 & $9 \cdot 22$ & $102 \cdot 04$ & 9.96 & 127.51 & 14.85 & 25.46 & $13 \cdot 1$ & 0.09 & -0.791 \\
\hline P-selectin (ng/ml) & $84 \cdot 16$ & $7 \cdot 17$ & 83.53 & $10 \cdot 42$ & -0.64 & 4.98 & $76 \cdot 23$ & $13 \cdot 22$ & 85.46 & 14.97 & $9 \cdot 23$ & 10.00 & 0.71 & -0.391 \\
\hline \multicolumn{15}{|l|}{$\mathrm{BP}$} \\
\hline Systolic BP (mmHg) & $126 \cdot 45$ & $2 \cdot 88$ & $122 \cdot 23$ & $2 \cdot 30$ & $-4 \cdot 23$ & 1.95 & 121 & 2.93 & $123 \cdot 70$ & $2 \cdot 66$ & $2 \cdot 34$ & 1.39 & 0.05 & $-1 \cdot 187$ \\
\hline Diastolic BP (mmHg) & $84 \cdot 24$ & $2 \cdot 37$ & $81 \cdot 39$ & $2 \cdot 37$ & -2.85 & 1.56 & $81 \cdot 819$ & $2 \cdot 21$ & 82.45 & $2 \cdot 16$ & 0.63 & 1.49 & 0.41 & -0.703 \\
\hline
\end{tabular}

$\Delta$, Week 16-week 0; HOMA-IR, homoeostasis model assessment of insulin resistance; hs-CRP, high-sensitivity C-reactive protein; ICAM-1, intercellular adhesion molecule 1.

${ }^{*} P$ value $<0.05$ for modification during the study (week $0 \mathrm{v}$. week 16) in each group.

$\dagger P$ values refer to differences between the energy restriction group and the control group.

$\ddagger$ Cohen's $d$ effect size.

however, the study conducted by Barnes et al. ${ }^{(37)}$ failed to show a statistically significant reduction in AHI in obese patients with mild-to-moderate sleep apnoea, after following a very low-energy diet combined with physical activity for 16 weeks, which resulted in an average weight loss of $12.3 \mathrm{~kg}$.

As expected, in the present study, the degree of weight loss was lower than that observed in clinical trials that evaluated the effects on OSA severity of weight loss induced by severe energy restriction $^{(9,34,36)}$ or severe energy restriction associated with increased physical activity ${ }^{(17,18,35)}$. Despite the lower weight loss observed in this study, the degree of improvement in parameters of OSA severity was similar to that observed in some randomised clinical trials in which participants presented a greater weight loss ${ }^{(17,18)}$. On the other hand, in some studies, a greater weight loss was achieved and was associated with higher improvements in parameters of OSA severity ${ }^{(\mathcal{Q}, 34)}$. Therefore, these findings suggest that other factors rather than absolute weight loss may interfere with the degree of improvement in OSA severity, such as, for example, the site of adiposity reduction (central $v$. peripheral).

Only uncontrolled studies addressed the impact of weight loss induced by moderate energy restriction, but they are not similar to the present investigation because energy restriction was coupled with physical activity ${ }^{(19,20)}$. These studies reported improvements 
Table 6. Correlations between changes in nutritional parameters and changes in the variables obtained during the sleep study, biochemical biomarkers, blood pressure (BP) and endothelial function

\begin{tabular}{|c|c|c|c|c|c|c|c|c|}
\hline & \multicolumn{2}{|c|}{$\Delta$ Body weight $(\mathrm{kg})^{\star}$} & \multicolumn{2}{|c|}{$\Delta$ Body fat $(\mathrm{kg})^{\star}$} & \multicolumn{2}{|c|}{$\Delta$ Waist circumference $(\mathrm{cm})^{\star}$} & \multicolumn{2}{|c|}{$\Delta$ Neck circumference $(\mathrm{cm})^{\star}$} \\
\hline & $r$ & $P$ & $r$ & $P$ & $r$ & $P$ & $r$ & $P$ \\
\hline$\Delta \mathrm{AHI}$ (events/h) & 0.55 & 0.03 & 0.50 & 0.05 & 0.60 & 0.01 & 0.43 & 0.09 \\
\hline$\Delta \mathrm{RDI}$ (events/h) & 0.60 & 0.01 & 0.58 & 0.02 & 0.67 & 0.005 & 0.56 & 0.02 \\
\hline$\Delta$ ODI (events/h) & 0.54 & 0.03 & 0.46 & 0.07 & 0.60 & 0.01 & 0.46 & 0.07 \\
\hline$\Delta$ Minimum $\mathrm{O}_{2}$ saturation (\%) & -0.46 & 0.07 & -0.47 & 0.07 & -0.52 & 0.04 & -0.47 & 0.07 \\
\hline$\Delta$ Noradrenaline $(\mathrm{pg} / \mathrm{ml})$ & 0.42 & 0.09 & 0.42 & 0.09 & 0.53 & 0.03 & 0.29 & 0.27 \\
\hline$\Delta$ Systolic BP (mmHg) & 0.71 & 0.001 & 0.70 & 0.002 & 0.68 & 0.003 & 0.61 & 0.009 \\
\hline$\Delta$ Diastolic BP $(\mathrm{mmHg})$ & 0.56 & 0.02 & 0.62 & 0.008 & 0.61 & 0.01 & 0.34 & 0.18 \\
\hline$\Delta$ Adiponectin $(\mu \mathrm{g} / \mathrm{ml})$ & -0.40 & 0.12 & -0.30 & 0.26 & -0.53 & 0.04 & -0.48 & 0.06 \\
\hline$\Delta$ Insulin $(\mu \mathrm{U} / \mathrm{ml})$ & 0.53 & 0.03 & 0.54 & 0.03 & 0.52 & 0.03 & 0.59 & 0.01 \\
\hline$\triangle \mathrm{HOMA}-\mathrm{IR}$ & 0.55 & 0.02 & 0.54 & 0.02 & 0.57 & 0.02 & 0.53 & 0.03 \\
\hline
\end{tabular}

$\Delta$, Week 16-week 0; AHI, apnoea/hypopnoea index; RDI, respiratory disturbance index; ODI, $\mathrm{O}_{2}$ desaturation index; HOMA-IR, homoeostasis model assessment of insulin resistance.

* After adjustments for confounding factors (age, sex and values of the variables involved in the analysis at baseline).

Table 7. Correlations between changes in variables obtained during the sleep study and the changes in biochemical biomarkers, blood pressure (BP) and endothelial function

\begin{tabular}{|c|c|c|c|c|}
\hline & \multicolumn{2}{|c|}{$\Delta \mathrm{AHI}$ (events/h) } & \multicolumn{2}{|c|}{$\begin{array}{l}\Delta \text { Minimum } \mathrm{O}_{2} \\
\text { saturation (\%) }\end{array}$} \\
\hline & $r$ & $P$ & $r$ & $P$ \\
\hline$\Delta$ Glucose $(\mathrm{mmol} / \mathrm{l})$ & 0.03 & 0.92 & -0.27 & 0.23 \\
\hline$\Delta$ Insulin $(\mu \mathrm{U} / \mathrm{ml})$ & 0.10 & 0.68 & -0.27 & 0.25 \\
\hline$\triangle \mathrm{HOMA}-\mathrm{IR}$ & 0.12 & 0.60 & -0.31 & 0.17 \\
\hline$\Delta$ Total cholesterol $(\mathrm{mmol} / \mathrm{l})$ & 0.04 & $0 \cdot 88$ & -0.17 & 0.47 \\
\hline$\Delta \mathrm{HDL}$-cholesterol (mmol/l) & 0.10 & 0.66 & -0.12 & 0.61 \\
\hline$\Delta \mathrm{LDL}$-cholesterol (mmol/l) & 0.07 & 0.77 & -0.17 & 0.45 \\
\hline$\triangle \mathrm{TAG}(\mathrm{mmol} / \mathrm{l})$ & $-0 \cdot 14$ & 0.56 & 0.02 & 0.92 \\
\hline$\Delta$ Adiponectin $(\mu \mathrm{g} / \mathrm{ml})$ & -0.39 & $0 \cdot 10$ & 0.54 & 0.01 \\
\hline$\Delta \mathrm{hs}-\mathrm{CRP}(\mathrm{ng} / \mathrm{dl})$ & 0.72 & 0.0005 & -0.57 & 0.008 \\
\hline$\Delta$ Adrenaline $(\mathrm{pg} / \mathrm{ml})$ & 0.03 & 0.89 & -0.09 & 0.70 \\
\hline$\Delta$ Noradrenaline $(\mathrm{pg} / \mathrm{ml})$ & 0.06 & 0.79 & 0.30 & 0.19 \\
\hline$\Delta \mathrm{ICAM}-1$ (ng/ml) & 0.12 & 0.64 & -0.42 & 0.08 \\
\hline$\Delta \mathrm{P}$-selectin $(\mathrm{ng} / \mathrm{ml})$ & -0.21 & 0.39 & 0.13 & 0.58 \\
\hline$\Delta \mathrm{RHI}$ & 0.05 & 0.84 & -0.20 & 0.37 \\
\hline$\Delta$ Systolic BP (mmHg) & 0.34 & $0 \cdot 15$ & -0.40 & 0.07 \\
\hline$\Delta$ Diastolic BP $(\mathrm{mmHg})$ & 0.03 & 0.91 & 0.02 & 0.93 \\
\hline
\end{tabular}

in OSA similar to that observed in our participants, after a modest weight loss similar to that observed in our participants.

Bariatric surgery is often touted as the most effective option for treating obesity and its co-morbidities, including $\mathrm{OSA}^{(38)}$. Dixon et $a l{ }^{(39)}$ evaluated whether surgically induced weight loss was more effective than conventional weight loss therapy in the management of OSA. Patients lost a mean of $5.1 \mathrm{~kg}$ in the conventional weight loss programme compared with $27.8 \mathrm{~kg}$ in the bariatric surgery group $(P=0 \cdot 001)$. The AHI decreased by $14 \cdot 0$ events/h in the conventional weight loss group and by 25.5 events/h in the bariatric surgery group. Surprisingly, bariatric surgery compared with conventional weight loss therapy did not result in a statistically greater reduction in $\mathrm{AHI}(P=0 \cdot 18)$, despite major differences in weight loss ${ }^{(39)}$.
According to all the studies cited above (including ours), it is possible to conclude that there is a great heterogeneity between them in relation to the effect of weight loss on the parameters of OSA severity. For example, in one study, weight loss of $12.3 \mathrm{~kg}$ was not associated with a significant decrease in $\mathrm{AHI}^{(37)}$, whereas in another study participants who had lost $>3 \%$ of their weight had a significant decrease in $\mathrm{AHI}^{(20)}$. The possible explanations for this heterogeneity may include differences in BMI and in OSA severity at the beginning of the study, sample size, characteristics of the participants such as age and sex, the site of preferential adiposity loss as previously commented and the association of physical activity with energy restriction. Exercise can directly change the structure of sleep ${ }^{(10)}$. In a randomised clinical trial that evaluated the impact of exercise on the OSA severity, it was observed that exercise, as compared with stretching, resulted in a significant AHI and ODI reduction in the absence of a significant decrease in body weight ${ }^{(40)}$.

In this study, the ERG compared with the CG did not achieve a significant improvement in CVD risk factors (blood pressure, inflammatory biomarkers, metabolic profile and endothelial function). A possible explanation to account for this lack of differences may rest in the small sample size, as there were medium-to-large effect sizes for the majority of these variables as well as a significant association between changes in parameters of nutritional status and modification in CVD risk factors.

One could also hypothesise that in the present study the moderate weight loss improved OSA severity but not CVD risk factors, because excessive adiposity is a risk factor for OSA by 'direct' or 'mechanical' mechanisms. These mechanisms include enlargement of soft tissue structures within and surrounding the airways and an excessive fat deposition in the tongue, soft palate and uvula and also under the mandible. In addition, lung volumes are markedly reduced in obese patients due to increased abdominal fat mass. Reduction of lung volume may decrease longitudinal tracheal traction forces and pharyngeal wall tension, predisposing to airway narrowing ${ }^{(8)}$. Conversely, the benefits of weight loss on CDV risk factors may be mediated by 'indirect' or 'metabolic' mechanisms that may require either a greater weight loss, a longer time of intervention or both. Therefore, during 
weight loss, the benefits for OSA severity may be obtained more easily than the benefits regarding CVD risk factors.

Evidence suggests that weight loss can reduce sympathetic activity in obese patients ${ }^{(41,42)}$. The present clinical trial is accordance with this fact, because modest weight loss resulted in significant reduction in plasma levels of adrenaline. In addition, there was a positive and significant association between changes in WC and the variations in plasma concentrations of noradrenaline, demonstrating the importance of central body fat in the modulation of sympathetic activity. The decrease in sympathetic activity observed in the ERG can also be attributed to the reduction in OSA severity.

We observed a strong association between changes in parameters of OSA severity (AHI, minimum $\mathrm{O}_{2}$ saturation) and modifications in hs-CRP levels, which was maintained even after adjusting for confounding factors. Therefore, in this study, it can be inferred that the changes in the various parameters of OSA severity had greater impact on the levels of hs-CRP than changes in body weight or WC during the study period. Evidence suggests that OSA patients have increased levels of several pro-inflammatory cytokines and of hs-CRP ${ }^{(43-46)}$.

In addition, a significant inverse association was found between changes in WC and modifications in serum adiponectin levels, supporting the role of central adiposity in the regulation of serum adiponectin concentrations.

There are several limitations to the present study. The main limitation is the evaluation of a small number of individuals. Type I error inflation was likely, given the high number of statistical comparisons

In summary, we found that a modest weight loss ( $\leq 5 \%$ of initial body weight) in obese subjects with OSA was able to improve OSA severity and sympathetic activity. However, it did not improve other CVD risk factors such as blood pressure and insulin resistance. As weight loss presented a significant correlation with the decrease in blood pressure and insulin resistance and as the effect sizes for the intergroup differences were rated as medium-to-large, it is possible that if weight loss had been greater or sample size larger a meaningful decrease in blood pressure and insulin resistance might have been documented.

\section{Conclusion}

The findings of this study suggest that in obese patients with OSA moderate energy restriction is able to reduce OSA severity and sympathetic nervous system activity, but not other CVD risk factors.

\section{Acknowledgements}

The authors express their sincere gratitude to Maria de Lourdes Guimarães Rodrigues, Débora Cristina Torres Valença, Sileia Nascimento and José Firmino Nogueira Neto.

The present study was supported by Fundação Carlos Chagas Filho de Amparo à Pesquisa do Estado do Rio de Janeiro (FAPERJ)

J. F. R. F., A. F. S. and M. R. S. T. K. contributed to the study conception and design; J. F. R. F., L. d. S. A., A. F. S. and M. R. S. T. K. contributed to data collection, assembly, analysis and interpretation of the data; S. E. K. contributed to data analysis and interpretation; J. F. R. F., L. d. S. A., S. E. K., M. R. S. T. K. contributed to the manuscript drafting and approval of the final version of the manuscript.

There are no conflicts of interest.

\section{References}

1. Lee W, Nagubadi S, Kryger MH, et al. (2008) Epidemiology of obstructive sleep apnea: a population-based perspective. Expert Rev Respir Med 2, 349-364.

2. Rakel RE (2009) Clinical and societal consequences of obstructive sleep apnea and excessive daytime sleepiness. Postgrad Med 121, 86-95.

3. Loke YK, Brown JW, Kwok CS, et al. (2012) Association of obstructive sleep apnea with risk of serious cardiovascular events: a systematic review and meta-analysis. Circ Cardiovasc Qual Outcomes 5, 720-728.

4. Gami AS, Olson EJ, Shen WK, et al. (2013) Obstructive sleep apnea and the risk of sudden cardiac death: a longitudinal study of 10,701 adults. J Am Coll Cardiol 62, 610-616.

5. Kendzerska T, Gershon AS, Hawker G, et al. (2014) Obstructive sleep apnea and risk of cardiovascular events and all-cause mortality: a decade-long historical cohort study. PLOS Med 11, e1001599.

6. Lévy P, Ryan S, Oldenburg O, et al. (2013) Sleep apnoea and the heart. Eur Respir Rev 22, 333-352.

7. Torres-Alba F, Gemma D, Armada-Romero E, et al. (2013) Obstructive sleep apnea and coronary artery disease: from pathophysiology to clinical implications. Pulm Med 2013, 768064.

8. Drager LF, Togeiro SM, Polotsky VY, et al. (2013) Obstructive sleep apnea. A cardiometabolic risk in obesity and the metabolic syndrome. J Am Coll Cardiol 62, 569-576.

9. Johansson K, Neovius M, Lagerros YT, et al. (2009) Effect of a very low energy diet on moderate and severe obstructive sleep apnoea in obese men: a randomized controlled trial. BMJ 339, b4609.

10. Shneerson J \& Wright J (2001) Lifestyle modification for obstructive sleep apnoea. The Cochrane Database of Systematic Reviews, issue 1, CD002875.

11. Epstein LJ, Kristo D, Strollo PJ, et al. (2009) Adult obstructive sleep apnea Task Force of the American Academy of Sleep Medicine: clinical guideline for the evaluation, management and long-term care of obstructive sleep apnea in adults. J Clin Sleep Med 5, 263-276.

12. Qaseem A, Holty JE, Owens DK, et al. (2013) Management of obstructive sleep apnea in adults: a clinical practice guideline from the American College of Physicians. Ann Intern Med 159, 471-483.

13. Jensen MD, Ryan DH, Apovian CM, et al. (2013) 2013 AHA/ACC/ TOS guideline for the management of overweight and obesity in adults: a report of the American College of Cardiology/American Heart Association Task Force on Practice Guidelines and The Obesity Society. Circulation 129, S102-S138.

14. Tsigos C, Hainer V, Basdevant A, et al. (2008) Obesity management Task Force of the European Association for the study of obesity. Management of obesity in adults: European Clinical Practice Guidelines. Obes Facts 1, 106-116.

15. Associação Brasileira de Estudos Sobre Obesidade (2009) Diretrizes brasileiras de obesidade 2009/2010 (Brazilian Guideline for Obesity 2009/2010). AC Farmacêutica 3.ed. Itapevi, SP, Brazil. http://www.abeso.org.br/pdf/diretrizes_ brasileiras_obesidade_2009_2010_1.pdf

16. Anandam A, Akinnusi M, Kufel T, et al. (2013) Effects of dietary weight loss on obstructive sleep apnea: a metaanalysis. Sleep Breath 17, 227-234. 
17. Foster GD, Borradaile KE, Sanders MH, et al. (2009) A randomized study on the effect of weight loss on obstructive sleep apnea among obese patients with type 2 diabetes. The Sleep AHEAD Study. Arch Intern Med 169, 1619-1626.

18. Tuomilehto HP, Seppä JM, Partinen MM, et al. (2009) Lifestyle intervention with weight reduction: first-line treatment in mild obstructive sleep apnea. Am J Respir Crit Care Med 179, 320-327.

19. Gilardini L, Lombardi C, Redaelli G, et al. (2013) Glucose tolerance and weight loss in obese women with obstructive sleep apnea. PLOS ONE 8, e61382.

20. Iguchi A, Yamakage H, Tochiya M, et al. (2013) Effects of weight reduction therapy on obstructive sleep apnea syndrome and arterial stiffness in patients with obesity and metabolic syndrome. J Atherosclerosis Thromb 20, 807-820.

21. Hainer V, Toplak H \& Mitrakou A (2008) Treatment modalities of obesity: what fits whom? Diabetes Care 31, S269-S277.

22. Baecke JA, Burema J \& Frijters JE (1982) A short questionnaire for the measurements of habitual physical activity in epidemiological studies. Am J Clin Nutr 36, 936-942.

23. Florindo AA, Latorre MRDO, Jaime PC, et al. (2004) Metodologia para a avaliação da atividade física habitual em homens com 50 anos ou mais (Methodology for the evaluation of habitual physical activity in men aged 50 years or more). Rev Saúde Pública 38, 1-7.

24. The National Academy Press (2005) Dietary reference intakes for energy, carbohydrate, fiber, fat, fatty acids, cholesterol, protein, and amino acids (macronutrients). http://www.nap.edu/books/ 0309085373/html/1324.html (accessed November 2011).

25. Yuceege M, Firat H, Demir A, et al. (2013) Reliability of the Watch-PAT200 ${ }^{\circledR}$ in detecting sleep apnea in highway bus drivers. J Clin Sleep Med 9, 339-344.

26. Weimin L, Rongguang W, Dongyan H, et al. (2013) Assessment of a portable monitoring device WatchPAT200 ${ }^{\circledR}$ in the diagnosis of obstructive sleep apnea. Eur Arch Otorhinolaryngol 270, 3099-3105.

27. Sichieri R \& Everhart JE (1998) Validity of a Brazilian frequency questionnaire against dietary recalls and estimated energy intake. Nutr Res 8, 1649-1659.

28. Stradling JR \& Crosby JH (1991) Predictors and prevalence of obstructive sleep apnoea and snoring in 1001 middle aged men. Thorax 46, 85-90.

29. Matthews DR, Hosker JP, Rudenski AS, et al. (1985) Homeostasis model assessment: insulin resistance and beta-cell function from fasting plasma glucose and insulin concentration in man. Diabetologia 28, 412-419.

30. Friedewald WT, Lévy RL \& Fredckson DS (1972) Estimation of the concentration of low-density lipoprotein cholesterol in plasma, without use of the preparative ultracentrifuge. Clin Chem 18, 499-502.

31. Sociedade Brasileira de Cardiologia/Sociedade Brasileira de Hipertensão/Sociedade Brasileira de Nefrologia (2010) VI Diretrizes Brasileiras de Hipertensão (Brazilian guideline for hypertension). Arq Bras Cardiol 95, 1-51.
32. Bonetti PO, Pumper GM, Higano ST, et al. (2004) Noninvasive identification of patients with early coronary atherosclerosis by assessment of digital reactive hyperemia. J Am Coll Cardiol 44, 2137-2141.

33. Cohen J (1992) A power primer. Psychol Bull 112, 155-159.

34. Kansanen M, Vanninen E, Tuunainen A, et al. (1998) The effects of a very low calorie diet-induced weight loss on the severity of obstructive sleep apnoea and autonomic nervous function in obese patients with obstructive sleep apnoea syndrome. Clin Physiol 4, 377-385.

35. Kajaste S, Brander PE, Telakivi T, et al. (2004) A cognitivebehavioral weight reduction program in the treatment of obstructive sleep apnea syndrome with or without initial nasal CPAP: a randomized study. Sleep Med 5, 125-131.

36. Nerfeldt P, Nilsson BY, Mayor L, et al. (2010) A two-year weight reduction program in obese sleep apnea patients. J Clin Sleep Med 6, 479-486.

37. Barnes M, Goldsworthy UR, Cary BA, et al. (2009) A diet and exercise program to improve clinical outcomes in patients with obstructive sleep apnea - a feasibility study. J Clin Sleep Med 5, 409-415.

38. Sarkhosh K, Switzer NJ, El-Hadi M, et al. (2013) The impact of bariatric surgery on obstructive sleep apnea: a systematic review. Obes Surg 23, 414-423.

39. Dixon JB, Schachter LM, O'Brien PE, et al. (2012) Surgical vs conventional therapy for weight loss treatment of obstructive sleep apnea: a randomized controlled trial. JAMA $\mathbf{3 0 8}$, 1142-1149.

40. Kline CE, Crowley EP, Ewing GB, et al. (2011) The effect of exercise training on obstructive sleep apnea and sleep quality: a randomized controlled trial. Sleep 34, 1631-1640.

41. Lambert EA, Rice T, Eikelis N, et al. (2014) Sympathetic activity and markers of cardiovascular risk in nondiabetic severely obese patients: the effect of the initial 10\% weight loss. Am J Hypertens 27, 1308-1315.

42. Lambert E, Straznicky NE, Dawood T, et al. (2011) Change in sympathetic nerve firing pattern associated with dietary weight loss in the metabolic syndrome. Front Physiol 26, $2-52$.

43. Shamsuzzaman AS, Winnicki M, Lanfranchi P, et al. (2002) Elevated C-reactive protein in patients with obstructive sleep apnea. Circulation 105, 2462-2464.

44. Yokoe T, Minoguchi K, Matsuo H, et al. (2003) Elevated levels of C-reactive protein and interleukin- 6 in patients with obstructive sleep apnea syndrome are decreased by nasal continuous positive airway pressure. Circulation 107, 1129-1134

45. Punjabi NM \& Beamer BA (2007) C-reactive protein is associated with sleep disordered breathing independent of adiposity. Sleep 30, 29-34.

46. Lui MM, Lam JC, Mak HK, et al. (2009) C-reactive protein is associated with obstructive sleep apnea independent of visceral obesity. Chest 135, 950-956. 